\title{
Seroprevalencia de la infección por el virus herpes simplex tipo 2 en tres grupos poblacionales de la Ciudad de México
}

\author{
Carlos J Conde-González, Q BP, M en C, Dr en $C$, ${ }^{(1)}$ Eduardo Lazcano-Ponce, MC, M en C, Dr en $C_{,}{ }^{(2)}$ \\ Carlos Hernández-G irón, $M C, M$ en $C$, ${ }^{(2)}$ Luis Juárez-Figueroa, $M C$, \\ Jennifer S Smith, MPH, PhD, ${ }^{(3)}$ Mauricio Hernández-Avila, MC, MPH, D Sc. ${ }^{(2)}$
} Conde-González CJ, Lazcano-Ponce E,
Hernández-Girón C, Juárez-Figueroa L, Smith JS, Hernández-Avila M.

Seroprevalencia de la infección por el virus herpes simplex tipo 2 en tres grupos poblacionales de la Ciudad de México. Salud Publica Mex 2003;45 supl 5:S608-S616. El texto completo en inglés de este artículo también está disponible en: http://www.insp.mx/salud/index.html

\section{Resumen}

Objetivo. D eter minar la sero prevalencia de infección por el virus herpes simplex tipo 2 y los factores epidemiologicos asociados a ella, en tres grupos de población femenina de la Ciudad de México. Material y métodos. Estudio transversal efectuado en el año 2000, que incluyó mujeres de la Ciudad de México diagnosticadas con cáncer de mama, cáncer cervical ,y mujeres de población general negativas al Papanicolaou. Todas las participantes proporcionaron su consentimiento informado para responder un cuestionario socio demográfico y de vida sexual, y permitir la toma de una muestra sanguínea. La presencia de anticuerpos contra el virus herpes simplex tipo 2 entre las mujeres se realizó por la técnica de "W estern blot" específica para el virus herpes simplex tipo 2; las aso ciaciones entre estos resultados y los datos de la encuesta se analizaron estadísticamente, de manera cruda y ajustada. Resultados. Las mujeres con cáncer cervical tuvieron una seroprevalencia de infección por el virus herpes simplex tipo 2 de 46.8\% (191/408); las de población general negativas al Papanicolaou de $29.3 \%$ (214/730), y aquellas con cáncer de mama de $22.6 \%$ (29/128). Las variables asociadas significativamente a la seropositividad contra este virus fueron la edad creciente, el aumento en el número de parejas sexuales, tener cáncer cervical, y entre las mujeres con esa patolo gía, el inicio antes de los 21 años de edad de la actividad sexual y
Conde-González CJ, Lazcano-Ponce E, Hernández-Girón C, Juárez-Figueroa L, Smith JS, Hernández-Avila M.

Herpes simplex virus type 2 seroprevalence among

three female population groups from Mexico City.

Salud Publica Mex 2003;45 supl 5:S608-S616.

The English version of this paper is available too at: http://www.insp.mx/salud/index.html

\begin{abstract}
A bstract
Objective To determine the seroprevalence of type 2 herpes simplex virus (HSV-2) infection and risk factors in three female population groups in Mexico City. Material and Methods A cross-sectional study was carried out in 2000 , among a sample po pulation of women living in Mexico City, diagno sed with breast or cervical cancer, and women with negative Pap test results from the general population. Informed consent was obtained from all participants to answer a sociodemographic and sex life questionnaire and draw a blood specimen. The presence of antibodies against type 2 herpes simplex virus was determined using the typespecific Western blot technique. Crude and adjusted statistical analyses were performed on the associations between survey data and infection outcomes. Results. W omen with cervical cancer had a sero prevalence of HSV2 infection of $46.8 \%$ (191/408); that of women from the general population who were Pap negative was 29.3\% (214/730). For women with breast cancer, the seroprevalence was $22.6 \%$ (29/128).Variables significantly associated with seropositivity against HSV-2 infection were older age, more sexual partners, having cervical cancer, and, among cervical cancer patients, beginning sexual activity before 21 years of age and being divorced or separated. Conclusions Study findings showed statistically significant differences in the prevalence of viral
\end{abstract}

(1) Dirección de Microbiología.C entro de Investigación sobre Enfermedades Infecciosas, Instituto N acional de Salud Pública (IN SP), C uernavaca, Morelos, México.

(2) Dirección de Epidemiología. Centro de Investigación en Salud Poblacional, IN SP. Cuernavaca, Morelos, México.

(3) Unit of Field and Intervention Studies. International A gency for Research on Cancer. Lyon, France.

Fecha de recibido: 11 de 0 ctubre 2002 • Fecha de aprobado: 23 de julio de 2003

Solicitud de sobretiros: Dr. Carlos J Conde-González. Dirección de Microbiología. Centro de Investigación sobre Enfermedades Infecciosas; Instituto N acional de Salud Pública. Avenida Universidad 655, colonia Santa María A huacatitlán, 62508 Cuernavaca, Morelos, México.

Correo electrónico: cjconde@ insp.mx 
el estar divorciadas o separadas. Conclusiones, Los hallazgos observados revelaron diferencias estadísticas en la seroprevalencia del virus herpes, de acuerdo con los grupos poblacionales estudiados. La frecuencia global de la infección viral entre las participantes las sitúa en un riesgo intermedio, en comparación con otros grupos poblacionales que en México son de alto y bajo riesgo (trabajadoras sexuales y estudiantes universitarias, respectivamente), analizados en años recientes. Las principales car acterísticas relacionadas al contacto con el virus herpes simplex tipo 2 para las subpoblaciones estudiadas correspondieron, como era de esperarse, a los antecedentes del estilo de vida sexual y a la probabilidad de infección que confiere la mayor edad. El texto completo en inglés de este artículo también está disponible en: http://www.insp.mx/salud/index.html

Palabras clave: virus herpes simplex tipo 2; sero prevalencia; población femenina;neoplasma del cuello uterino;neoplasma de la mama; México infection by population gro ups. The overall frequency of viral infection among study subjects shows that this is an intermediate risk population, as compared with other population groups in Mexico at high (sexual workers) and low (college students) risk. The main characteristics associated with exposure to type 2 herpes simplex virus for the subpopulations corresponded, as expected, to sexual behavior and to the probability of infection with older age. The English version of this paper is available too at: http:// www.insp.mx/salud/index.html

Key words: type 2 herpes simplex virus; seroprevalence; female population; cervical neoplasia; mammary neoplasia; Mexico
E 1 virus herpes simplex pertenece a los ADN virus, forma parte de la familia Herpesviridae y se clasifica en dos tipos: el virus herpes simplex tipo 1 (VHS-1) asociado mayoritariamente a lesiones faciales y bucales; y el virus herpes simplex tipo 2 (VHS-2), asociado principalmente a lesiones genitales. ${ }^{1}$ De hecho, el VHS2 es uno de los agentes transmitidos por vía sexual más frecuentes en el humano, y aun cuando la infección genital también puede ser ocasionada por el VHS-1, la historia natural de uno u otro tipo viral es diferente. El agente causal en la mayoría de las infecciones genitales es el VHS-2 y aproximadamente en 15\% de ellas se encuentra el VHS-1, lo cual se ha relacionado con la

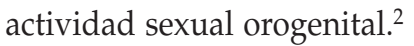

A su vez, la prevalencia de marcadores serológicos de infección por el VHS-2 varía de acuerdo con el tipo de población estudiada. En trabajadoras sexuales de diversos países las seroprevalencias son consistentemente mayores a las observadas en grupos femeninos que acuden a planificación familiar o a clínicas de detección de enfermedades venéreas. ${ }^{3}$ En la Ciudad de México, por ejemplo, la prevalencia de marcadores serológicos de infección por VHS-2 se han encontrado de $65.1 \%$ y $60.8 \%$ en trabajadoras sexuales, y de $18.1 \%$ en asistentes a consulta ginecológica. ${ }^{4-6}$

Por otra parte, las estadísticas oficiales de la Secretaría de Salud en nuestro país indican que la tasa de incidencia del herpes genital diagnosticado clínicamente, ha estado alrededor de los siete casos por 100000 habitantes durante el año 2001.*

\footnotetext{
* Información disponible en: www.salud.gob.mx/unidades/conasida

En relación con el diagnóstico serológico del VHS-2, las pruebas para detección de anticuerpos son útiles para establecer la historia de la exposición a la infección por el virus. Para fines de estudios seroepidemiológicos la técnica de "Western blot" (inmunoelectrotransferencia) tipo viral específica ha demostrado su valor en la determinación de las características de las poblacio-nes asociadas a la infección por el VHS-2. ${ }^{7-10}$

Con el propósito de abundar en el conocimiento de la seroepidemiología del VHS-2 en nuestro ámbito, como un agente transmitido por contacto sexual, se realizó este estudio en tres grupos de población femenina en la Ciudad de México; dos de ellos, con patología oncogénica, y el otro, representativo de la población general, para determinar la prevalencia del VHS-2 y los factores asociados con la infección.

\section{Material y métodos}

Se realizó un estudio epidemiológico de seroprevalencia del VHS-2 durante el año 2000, en tres grupos poblacionales de mujeres de la Ciudad de México. Para ello se diseñó un estudio transversal, considerando tres bases poblacionales: mujeres diagnosticadas con cáncer de mama, cáncer cervical y mujeres de población general.

\section{Población general}

Entre mayo de 1994 y diciembre de 1996 se seleccionaron 730 mujeres mediante muestreo probabilístico estratificado por edad en aquellas residentes, al menos por dos años, en el área metropolitana de la Ciudad de México. Se identificaron 3694 hogares seleccionados aleatoriamente del Marco Muestral de la Dirección General de 
Epidemiología, de la Secretaría de Salud (SSA), de donde se obtuvieron 730 y se entrevistó a una mujer con prueba de Papanicolaou negativa por cada uno de ellos, en edad reproductiva (15-49 años), y a las cuales se les administró un cuestionario que incluía variables sociodemográficas, ginecobstétricas y de comportamiento sexual. $^{11}$

\section{Casos de cáncer de mama y cáncer cervical}

Entre septiembre de 1990 y diciembre de 1995 se identificaron casos incidentes de cáncer de mama (128) y cáncer cervical (408), en ocho hospitales de la Ciudad de México: cuatro hospitales de atención a población derechohabiente: Hospital de Ginecología No. 3 y No. 4 del Instituto Mexicano del Seguro Social, Hospital 20 de Noviembre del Instituto de Seguridad y Servicios Sociales de los Trabajadores del Estado, Centro Médico Militar; dos hospitales de atención a población no derechohabiente: Hospital General y Hospital de la Mujer de la SSA; y dos hospitales privados: Hospital de México y Hospital Metropolitano. Los casos fueron seleccionados de mujeres que acudían a atención ginecológica, confirmados histológicamente (biopsia). Adicionalmente se aplicó el mismo cuestionario sobre variables sociodemográficas, ginecobstétricas y de comportamiento sexual. Mayores detalles sobre el diseño e instrumentación de la investigación inicial ya han sido publicados con anterioridad. ${ }^{12,13}$

\section{Pruebas de laboratorio}

Previo consentimiento informado, se obtuvo una muestra de $10 \mathrm{ml}$ de sangre por punción venosa con el sistema Vacutainer (Becton-Dickinson, Estados Unidos de América (EUA), de cada mujer estudiada. Las muestras de suero fueron transferidas a viales etiquetados con código de barras y se mantuvieron congeladas a $-20{ }^{\circ} \mathrm{C}$ hasta su uso en el laboratorio. Para la determinación de anticuerpos anti-VHS-2 se realizó una prueba de inmunoelectrotransferencia utilizando como antígeno la proteína recombinante gG2 del VHS-2. ${ }^{14}$ Brevemente se infectaron células Sf9 con el baculovirus recombinante $\mathrm{AcDSMgG}-2$, luego se procedió a electroforesis en geles de poliacrilamida y electrotransferencia a hojas de nitrocelulosa. Dichas hojas se colocaron en dispositivos Multiscreen (Bio-Rad, EUA) para lograr la detección de anticuerpos anti-VHS-2, con un control positivo y uno negativo, simultáneamente en cada hoja (figura 1). Se consideraron positivas las muestras que dieron una banda coloreada con PM aparente de $118 \mathrm{Kda} .{ }^{15}$

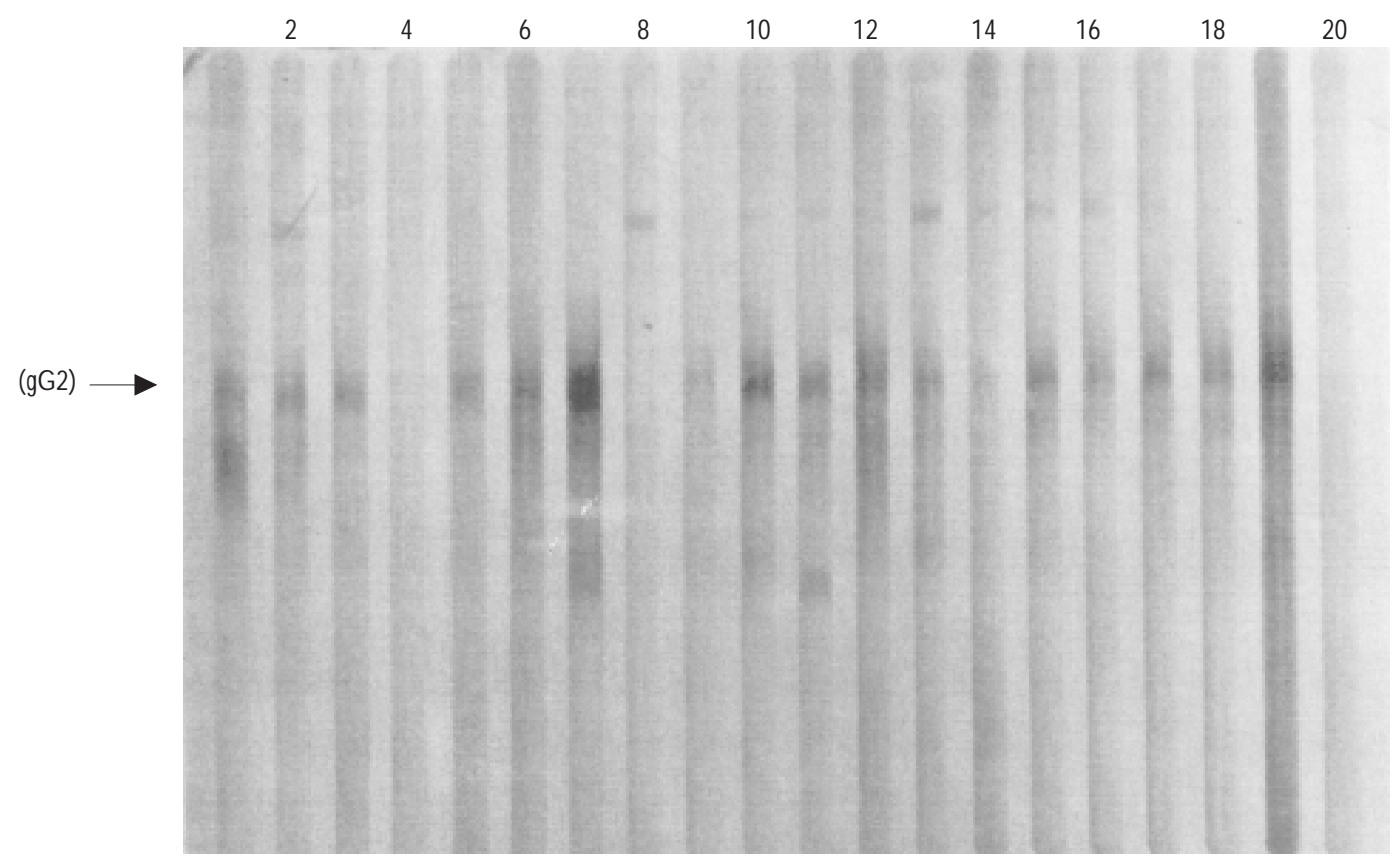

Figura 1. Prueba de W estern Blot tipo específica para VhS-2 Carril 10: control positivo. Carril 20: control negativo. Ejm: Carril 11-positivo, Carril 8-negativo. Ciudad de México, 2000. 


\section{A nálisis estadístico}

El análisis estadístico se realizó utilizando el paquete Stata. Un análisis univariado se elaboró para todas las variables, y se consideraron los tres grupos poblacionales por separado (cáncer de mama, cáncer de cérvix y población general), y, en conjunto, las variables continuas y categóricas. Se consideró como variable dependiente la prevalencia de VHS-2 en los tres grupos, y como variables independientes las características sociodemográficas, ginecológicas, y de comportamiento sexual, entre otras. También se construyó un índice de nivel socioeconómico, apoyado en la metodología propuesta por Bronfman y colaboradores, ${ }^{16}$ incluyendo variables de características de la vivienda: número de personas que viven en la casa, número de cuartos por vivienda, disposición de agua potable, luz eléctrica y drenajes, así como nivel socioeconómico del jefe de familia. Como medida de asociación epidemiológica se utilizó la razón de momios de prevalencias con sus respectivos intervalos de confianza a $95 \%$. Para ello se construyeron modelos de regresión logística simple y ajustada, entre la seroprevalencia del VHS-2 y los factores de riesgo, según grupo poblacional. Se realizó análisis logístico multivariado para controlar las posibles variables confusoras (edad, nivel de escolaridad, nivel socioeconómico, número de parejas sexuales y edad de inicio de vida sexual).

\section{Resultados}

La prevalencia de anticuerpos anti-VHS-2 en la población de estudio fue mayor en mujeres con cáncer cervical con 46.8\% (191/408), en relación con mujeres de población general negativas al Papanicolaou, 29.3\% (214/730) y mujeres con cáncer de mama, 22.6\% (29/128). Estas diferencias son estadísticamente significativas (valor $p=0.000$ ). Según grupo de edad, se observó un incremento en la seroprevalencia de VHS-2 en las mujeres de 35 y más años, para los tres grupos poblacionales (figura 2).

La media de edad fue mayor en las mujeres infectadas por VHS-2 para los tres grupos: mujeres de población general negativas al Papanicolaou 52.8 vs 45.0 años (infectadas vs no infectadas); aquellas con cáncer cervical 52 vs 44.7 años; y otras con cáncer de mama 54.3 vs 49.6 años. Para dos grupos la media de edad de inicio en la vida sexual activa fue menor en el grupo de seropositivas al VHS-2: mujeres con cáncer cervical 19.2 vs 20.1 años; y aquellas con cáncer de mama 21.1 vs 23.2 años. Estas diferencias fueron estadísticamente significativas. Para los 3 grupos la media de embarazos en toda su vida también fue mayor en las mujeres infectadas por VHS-2: las de población general negativas al Papanicolaou 6.2 vs 5.4 embarazos; aquellas con cáncer cervical 6.8 vs. 6.0 embarazos; y mujeres con cáncer de mama 5.0 vs. 4.4 embarazos. Estas diferencias son estadísticamente significativas entre los grupos ("Scheffé test, comparisson between groups"); si bien es cierto que los antecedentes ginecológicos están relacionados con la edad de las mujeres.

La seroprevalencia de VHS-2 se incrementó en forma lineal con la edad. Así, la razón de momios fue mayor en las mujeres de 45 y más años de edad, valores estadísticamente significativos en el grupo de las de población general (RM: 6.2 IC 95\% 3.4-11.5) y mujeres con cáncer cervical (RM: 4.6 IC 95\% 2.4-8.7), respecto al grupo de mujeres con cáncer de mama. No hubo diferencias estadísticamente significativas, según estado civil, nivel socioeconómico y escolaridad (cuadros I y II).

El número de partos vaginales fue un factor de asociación importante para la seropositividad al VHS-2 en el grupo de mujeres con cáncer cervical (2-4 partos RM: 2.5 IC 95\% 1.2-5.6), aunque no mostró una tendencia positiva. En el grupo de población general el número de parejas en toda la vida, también mostró mayor asociación con la exposición a herpes tipo 2, (RM: 2.8 IC 95\%: 1.8-4.6) y con cáncer cervical (RM: 2.1 IC 95\%: 1.4-3.1).

Se encontró que, conforme disminuía la edad de inicio de vida sexual, aumentaba la seroprevalencia de infección por VHS-2. Aunque estas diferencias no son

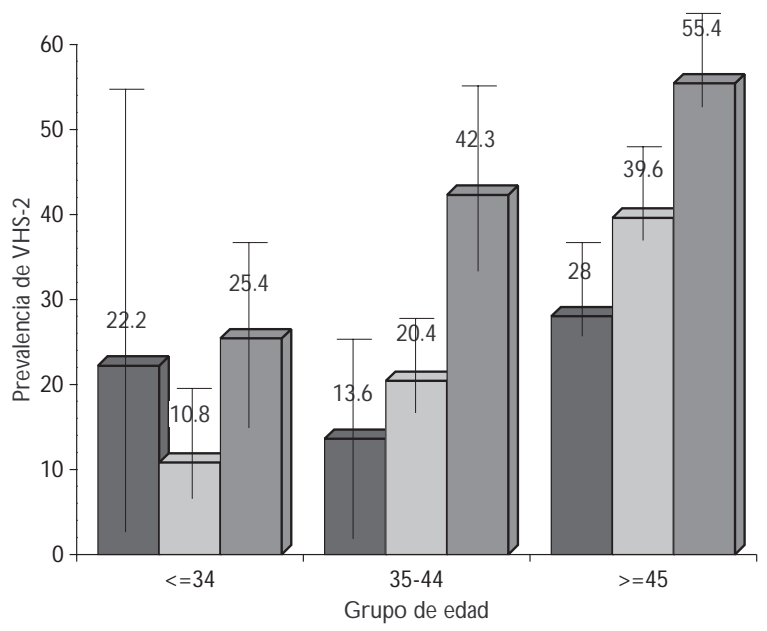

Cáncer de mama Población general Cáncer cervical

Figura 2. Seroprevalencia de Virus herpes simpleX tipo 2 según población y edad. CiUdad de México, 2000 
estadísticamente significativas, sí se observa una tendencia positiva en el grupo de cáncer de cérvix (cuadros I y II).

Estratificando por grupo de edad y número de parejas sexuales en toda su vida, las mujeres mayores de 45 años y con dos o más parejas sexuales, en los grupos de población general (RM: 20.4 IC 95\% 8.3-50.4) y con cáncer cervical (RM: 8.6 IC95\% 3.5-20.8) incrementaron significativamente su probabilidad de seropositividad al virus (cuadro III).

\section{Discusión}

Respecto a la sero-epidemiología de la infección por el VHS-2 en población mexicana este estudio es una contribución de relevancia en el medio local, toda vez que en los años recientes diversos trabajos sobre la seroprevalencia del VHS-2 en grupos poblacionales con riesgos variables de ITS han sido publicados. ${ }^{4,7,17-19} \mathrm{De}$ ellos, la mayoría ha carecido de representatividad poblacional y, en este caso particular, se presenta una

\section{Cuadro I}

Prevalencia de infección por VHS-2, según características socioeconómicas, ginecoobstétricas y de comportamiento sexual, en 3 grupos de mujeres. Ciudad de México, 2000

\begin{tabular}{|c|c|c|c|c|c|c|}
\hline & & & & & & \\
\hline & $\mathrm{N}$ & VHS-2 \% & $\mathrm{N}$ & VHS-2 \% & $\mathrm{N}$ & VHS-2 \% \\
\hline Edad (años) & & & & & & \\
\hline$<=34$ & 130 & 10.8 & 9 & 22.2 & 71 & 25.4 \\
\hline $35-44$ & 196 & 20.4 & 44 & 13.6 & 104 & 42.3 \\
\hline$>=45$ & 404 & 39.6 & 75 & 28.0 & 233 & 55.4 \\
\hline Prob.Chi & & 0.000 & & 0.195 & & 0.000 \\
\hline Escolaridad (años) & & & & & & \\
\hline$>=7$ & 50 & 16.0 & 11 & 18.2 & 10 & 50.0 \\
\hline $1-6$ & 520 & 26.6 & 70 & 24.3 & 240 & 44.2 \\
\hline Cero & 160 & 32.5 & 47 & 21.3 & 158 & 50.6 \\
\hline Prob.Chi ${ }^{2}$ & & 0.133 & & 0.617 & & 0.005 \\
\hline Estado civil & & & & & & \\
\hline Casada & 514 & 24.9 & 81 & 22.2 & 210 & 37.6 \\
\hline Soltero & 38 & 31.6 & 18 & 11.1 & 19 & 31.6 \\
\hline Unión Libre & 61 & 44.3 & 6 & 33.3 & 57 & 54.4 \\
\hline Divorciada/Viuda & 115 & 40.9 & 22 & 31.8 & 120 & 62.5 \\
\hline Prob.Chi & & 0.000 & & 0.423 & & 0.000 \\
\hline $\mathrm{N}$ ivel socioeconómico & & & & & & \\
\hline Alto & 123 & 32.5 & 29 & 27.6 & 13 & 49.2 \\
\hline Mediano & 405 & 27.6 & 46 & 21.7 & 174 & 47.1 \\
\hline Bajo & 202 & 30.7 & 53 & 20.8 & 96 & 43.8 \\
\hline Prob.Chi & & 0.513 & & 0.766 & & 0.649 \\
\hline No. embarazos & & & & & & \\
\hline $0-1$ & 53 & 32.1 & 26 & 23.1 & 22 & 27.3 \\
\hline $2-4$ & 292 & 24.3 & 57 & 17.5 & 127 & 41.7 \\
\hline $5-7$ & 193 & 29.0 & 22 & 31.8 & 110 & 51.8 \\
\hline$>=8$ & 192 & 36.5 & 23 & 26.1 & 149 & 50.3 \\
\hline Prob.Chi & & 0.038 & & 0.560 & & 0.088 \\
\hline No. partos vaginales & & & & & & \\
\hline $0-1$ & 137 & 31.4 & 44 & 18.2 & 50 & 26.0 \\
\hline $2-4$ & 281 & 24.6 & 46 & 21.7 & 144 & 45.1 \\
\hline $5-7$ & 170 & 29.4 & 20 & 35.0 & 120 & 54.2 \\
\hline$>=8$ & 142 & 34.6 & 18 & 22.2 & 94 & 51.1 \\
\hline Prob.Chi ${ }^{2}$ & & 0.071 & & 0.519 & & 0.007 \\
\hline No. abortos & & & & & & \\
\hline $\mathrm{N}$ inguno & 478 & 27.6 & 76 & 21.1 & 209 & 44.0 \\
\hline Uno & 125 & 24.0 & 34 & 26.5 & 96 & 46.9 \\
\hline$>=2$ & 127 & 40.9 & 18 & 22.2 & 103 & 52.4 \\
\hline Prob.Chil & & 0.005 & & 0.820 & & 0.375 \\
\hline
\end{tabular}

Continúa 
Continuación

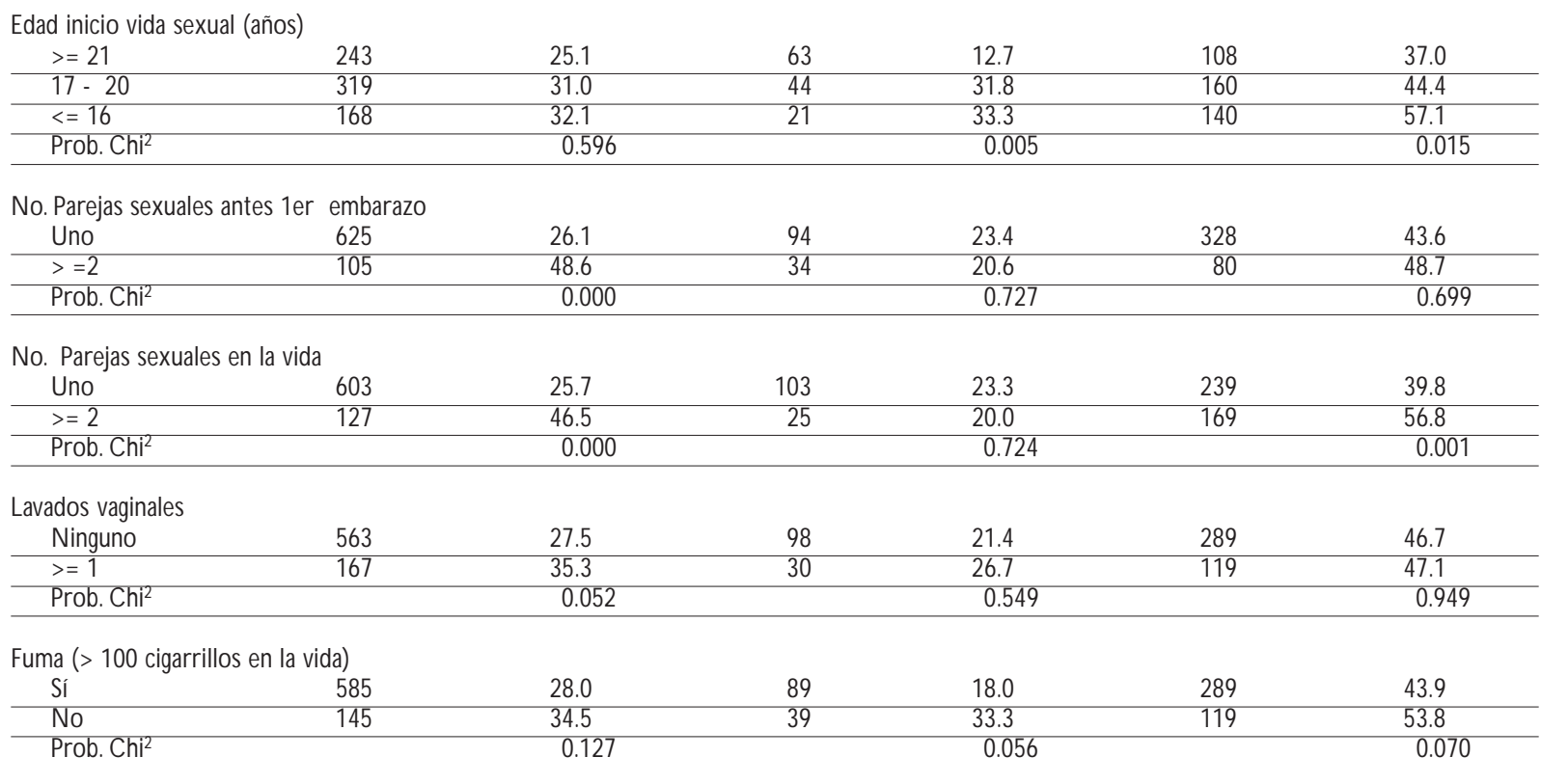

\section{Cuadro II}

Factores de riesgo para infección por VhS-2 según grupo de mujeres. Ciudad de México, 2000

\begin{tabular}{|c|c|c|c|c|c|c|}
\hline & & se poblacional & & cer de mama & & cer cervical \\
\hline & RM* & $95 \%$ IC & $\mathrm{RM}^{*}$ & $95 \%$ IC & RM* & $95 \%$ IC \\
\hline Edad (años) & & & & & & \\
\hline$<=34$ & 1 & & 1 & & 1 & \\
\hline $35-44$ & 2.4 & $1.2-4.6$ & 0.7 & $0.1-4.7$ & 2.6 & $1.3-5.3$ \\
\hline$>=45$ & 6.2 & $3.4-11.5$ & 1.7 & $0.3-10.0$ & 4.6 & $2.4-8.7$ \\
\hline Prueba de tendencia & $<0.05$ & & $<0.05$ & & $<0.05$ & \\
\hline Escolaridad (años) & & & & & & \\
\hline$>=7$ & 1 & & 1 & & 1 & \\
\hline $1-6$ & 2.0 & $0.8-4.7$ & 1.7 & $0.8-3.6$ & 2.0 & $0.9-4.7$ \\
\hline Cero & 1.5 & $0.6-3.7$ & 1.2 & $0.2-4.0$ & 1.5 & $0.6-3.7$ \\
\hline Prueba de tendencia & $>0.05$ & & $>0.05$ & & $>0.05$ & \\
\hline Estado civil & & & & & & \\
\hline Casada/ & 1 & & 1 & & 1 & \\
\hline Soltera & 1.2 & $0.6-2.7$ & 1.0 & $0.2-7.0$ & 0.6 & $0.2-1.8$ \\
\hline Unión libre & 2.3 & $1.2-4.3$ & 1.8 & $0.3-12.0$ & 1.6 & $0.8-3.1$ \\
\hline Divorciada/Separada & 1.5 & $0.9-2.4$ & 1.5 & $0.4-5.3$ & 2.2 & $1.3-3.8$ \\
\hline Prueba de tendencia & $>0.05$ & & $>0.05$ & & $<0.05$ & \\
\hline N ivel socieconómico & & & & & & \\
\hline Alto & 1 & & 1 & & 1 & \\
\hline Mediano & 0.8 & $0.5-1.3$ & 0.7 & $0.2-2.2$ & 1.0 & $0.6-1.6$ \\
\hline Bajo & 1.0 & $0.6-1.7$ & 1.0 & $0.3-3.3$ & 1.0 & $0.6-1.8$ \\
\hline Prueba de tendencia & $>0.05$ & & $>0.05$ & & $>0.05$ & \\
\hline No. embarazos & & & & & & \\
\hline $0-1$ & 1 & & 1 & & 1 & \\
\hline $2-4$ & 0.6 & $0.3-1.3$ & 0.9 & $0.3-2.9$ & 1.4 & $0.5-4.1$ \\
\hline $5-7$ & 0.4 & $0.2-1.0$ & 1.4 & $0.4-5.8$ & 1.5 & $0.5-4.4$ \\
\hline$>=8$ & 0.5 & $0.2-1.1$ & 0.3 & $0.1-1.7$ & 0.8 & $0.3-2.6$ \\
\hline Prueba de tendencia & $>0.05$ & & $>0.05$ & & $>0.05$ & \\
\hline
\end{tabular}


Continuación

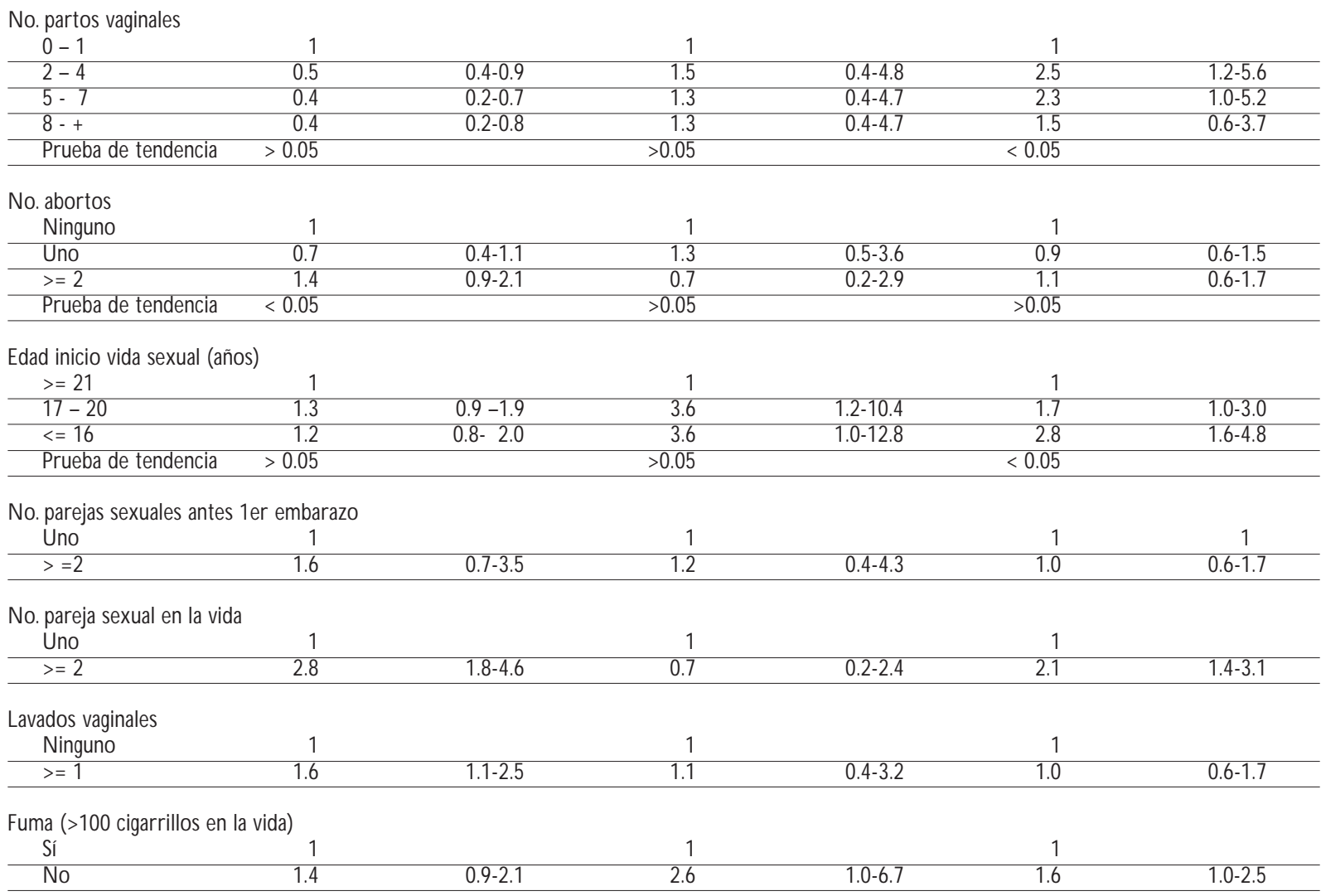

* RM ajustada por: edad, escolaridad, número de parejas sexuales y edad de inicio de vida sexual.

\section{Cuadro III \\ Razón de MOMIOS (RM) de INFECCIÓN POR VHS-2, ESTRATIFICADO POR EDAD Y NÚMERO de parejas sexuales, segun grupo poblacional. Ciudad de México, 2000}

\begin{tabular}{|c|c|c|c|c|c|c|}
\hline \multirow[b]{2}{*}{ Grupo de edad } & \multicolumn{4}{|c|}{ Mujeres con base poblacional } & \multicolumn{2}{|c|}{$\geq 2$ Parejas sexuales } \\
\hline & No. & RM & IC $95 \%$ & No. & RM & IC $95 \%$ \\
\hline$\leq 34$ Años & 106 & 1.0 & - - & 24 & 5.8 & $1.8-18.7$ \\
\hline $35-44$ & 164 & 3.1 & $1.3-7.5$ & 32 & 6.4 & $2.2-18.6$ \\
\hline$\geq 45$ Años & 333 & 7.8 & $3.5-17.4$ & 71 & 20.4 & $8.3-50.4$ \\
\hline
\end{tabular}

Prueba de tendencia $=p<.001$

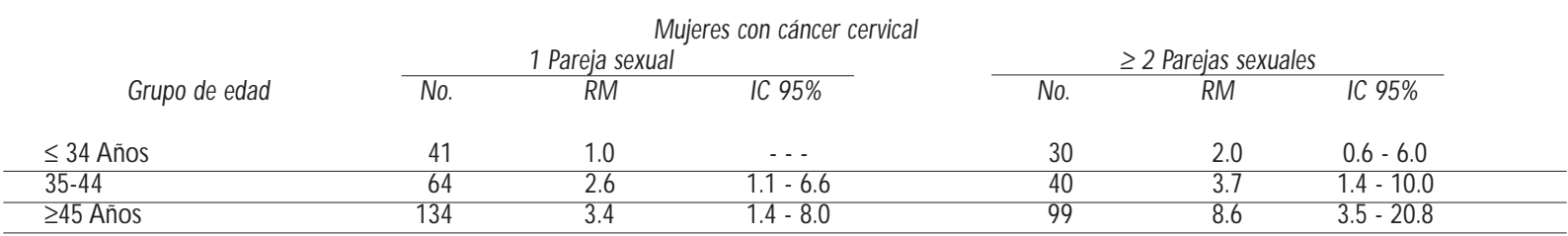

Prueba de tendencia $=p<.001$ 
combinación de dos subpoblaciones: la primera con patología oncológica seleccionada consecutivamente por conveniencia, y una más de base poblacional aleatoriamente seleccionada.

Un hallazgo central de esta investigación ha sido el demostrar una mayor seroprevalencia de anticuerpos contra el VHS-2 en mujeres con cáncer cervicouterino, respecto de aquellas con cáncer de mama y las de población general. Por otra parte, se confirmaron las observaciones de estudios previos en México ${ }^{4,7,17-19}$ y en el extranjero ${ }^{8,9,20,21}$ sobre las asociaciones entre la infección por el VHS-2 y marcadores de conducta sexual (edad de inicio de relaciones, número de parejas sexuales) y sociodemográficos (la edad). Cabe mencionar, a propósito de las características sociodemográficas de las participantes, que según se observa en la figura 2 el dato de una seroprevalencia anti-VHS-2 aparentemente mayor en el grupo de menor edad para los casos de cáncer de mama se debió posiblemente al pequeño tamaño de la muestra $(N=9)$ en ese subgrupo.

Al comparar las seroprevalencias anti-VHS-2 de las mujeres de población general $(29.3 \%)$ y con cáncer de mama $(22.6 \%)$ aquí analizadas con otros estudios en México, encontramos que el valor no difiere significativamente del resultado que otros trabajos han documentado en grupos seleccionados de mujeres asistentes a una clínica de ITS en el Distrito Federal, ${ }^{17}$ y al servicio ginecológico de un hospital de Cuernavaca, Morelos, ${ }^{6}$ prevalencias de $29.3 \%$ y $28.4 \%$, respectivamente.

En cambio, al contrastar con personas de mucho mayor riesgo para ITS como son las trabajadoras sexuales en la misma Ciudad de México, las frecuencias de infección por el VHS-2 son mayores, como era de esperarse, y se ubican en torno a $60 \%{ }^{4,7}$ Sin embargo, este último dato no difiere en gran medida de la seroprevalencia de $46.8 \%$ del subgrupo de mujeres con cáncer cervicouterino, quienes en este caso tendrían el antecedente de riesgo muy particular de haber sufrido una infección venérea por VPH oncogénico, misma que al interior de ese subgrupo podría explicarse por los antecedentes de un inicio de vida sexual más temprano y una tendencia hacia un mayor número de parejas sexuales a lo largo de su vida, en relación con los otros dos grupos estudiados.

El diseño de este estudio no permite en realidad establecer ninguna causalidad entre el hecho de la infección por VHS-2 y la infección por VPH manifestada ya como cáncer, puesto que no se puede conocer la temporalidad de los eventos y el contacto preciso de la transmisión viral. No obstante, es plausible reconocer que los estilos de vida y conducta sexual de las mujeres sean las condicionantes que marquen los riesgos al interior de un grupo dado para la adquisición y transmisión de las ITS comentadas.

La variable del grado de escolaridad que no se encontró asociada con la seroprevalencia de anticuerpos anti-VHS-2 difiere de otros estudios en México y los $\mathrm{EUA}^{6},{ }^{6,922}$ donde una menor escolaridad corresponde a mayores seroprevalencias. En nuestro caso, la observación discordante pudiera deberse a algunas condiciones subyacentes a los subgrupos estudiados que no fue posible explorar a través de la encuesta aplicada o la distribución azarosa de los niveles educativos.

En cuanto a la certeza de los diagnósticos de laboratorio en este trabajo, debe asentarse que para la identificación de los anticuerpos específicos contra el VHS-2 se usó un método estandarizado y validado de inmunoelectrotransferencia ${ }^{15}$ que utiliza como antígeno la glicoproteína 2 exclusiva de la envoltura del VHS-2. Así, esta técnica ha demostrado ser más sensible y específica que las pruebas de ensayo inmunoenzimáti$\mathrm{Co}^{23,24}$ además de que su logística de aplicación permite su utilización exitosa en estudios epidemiológicos como el presente, así como en investigaciones de posible aplicación clínica; por ejemplo, en la protección de recién nacidos de madres infectadas..$^{15}$ Otro hecho a considerarse sobre la técnica aplicada es que nuestro grupo la ha empleado consistentemente en lo que ya es una serie de estudios en diferentes grupos poblacionales del país que han incluido mujeres, hombres, adolescentes y universitarios. . $^{4,717-19,25-27,28}$

Como en todos los estudios observacionales de corte transversal, no es posible establecer asociaciones causa-efecto y los resultados obtenidos sólo pueden extrapolarse al interior de los grupos estudiados. Sin embargo, las mujeres participantes, además de los entrevistadores, no conocían la hipótesis de estudio, por lo que el sesgo de información ha sido minimizado.

Por último, con este estudio se ratifica la utilidad de practicar la serología del VHS-2 en grupos poblacionales sexualmente activos, como un estimador confiable del comportamiento sexual de los individuos que puede colocarles en riesgo de adquirir esta importante infección de transmisión sexual. ${ }^{7,29}$

\section{Referencias}

1.W hite D, Fenner F. Medical Virology. $4^{\text {th }}$ Ed. N ew York:A cademic Press, USA. 1996: 317-330.

2. Stanberry LR, Jorgensen D M, N ahmias AJ. Herpes simplex viruses 1 and 2. In: Evans AS, Kaslow RA, Ed.Viral infections of humans. 
Epidemiology and Control. $4^{\text {th }}$ Ed., N ew York: Plenum Medical Book Company. 1997: 419-454.

3. $\mathrm{N}$ ahmias AJ, Lee FK, Beckman-N ahmias S. Seroepidemiological and sociological patterns of Herpes simplex virus infection in the world. Scand J Infect D is 1990; 69(Suppl): 19-36.

4. U ribe-Salas F, Hernández-Avila M, Juárez-Figueroa L, C onde-G lez CJ, U ribe-Zúniga P: Risk factors for HSV type 2 infection among female CSW in Mexico City. Int J STD AIDS 1999; 10: 105-111.

5. Conde-G lez CJ. Enfermedades de transmisión sexual., México, DF: Intersistemas, 1999.

6. Zamilpa-Mejía LG, U ribe-Salas F, Juárez-Figueroa L, C alderón-Jaimes E, Conde-González CJ. Prevalencia y factores asociados para sífilis y herpes genital en dos grupos de población femenina. Salud Publica Mex 2003; 45 (supl)5:S617-S623.

7. C onde-G lez CJ, Juárez-Figueroa L, U ribe-Salas F, Hernández-N evárez $P$, Schmid DS, Calderón E et al:A nalysis of herpes simplex virus 1 and 2 infection in women with high risk sexual behaviour in Mexico. Int J Epidemiol 1999; 28: 571-576.

8. Cowan FM, Johnson AM, A shley R, Corey L, Mindel A. Antibody to herpes simplex virus type 2 as serological marker of sexual lifestyle in populations. Br Med J 1994; 309: 1325-1329.

9. W ald A, Koutsky L,A shley RL, Corey L. Genital herpes in a primary care clinic. D emographic and sexual correlates of herpes simplex type 2 infections. Sex Transm D is 1997; 24: 149-155.

10. D ada AJ, A jayi AO , D iamondstone L, Q uinn TC, Blattner W A, Biggar RJ. A serosurvey of Haemophilus ducreyi, syphilis and herpes simplex virus type 2 and their association with human immunodeficiency virus among female sex workers in Lagos, $N$ igeria. Sex Transm D is 1998; 25 : 237-242.

11. Hernández-Avila M, Lazcano E, Berumen J, Cruz A,Alonzo P, González L. Human papillomavirus 16-18 infection and cervical cancer in Mexico:A case-control study.Arch Med Res 1997; 28: 265-271. 12. Romieu I, Hernández M, Lazcano E, López L. Breast cancer and lactation history in Mexican women. Am J Epidemiol 1996;143: 543-552. 13. Lazcano-Ponce E, Hernández-A vila M, López-Carrillo L,Alonso de Ruiz P, G onzález-Lira G, Torres-Lobatón A et al. Factores de riesgo reproductivo e historia de vida sexual asociados a cáncer cervical en una muestra de mujeres de la C d. de México. Rev Invest C lin 1995;47: 377-385.

14. Sánchez D, Pellet PE. Expression of HSV-1 and HSV-2 glycoprotein G in insect cells by using a novel baculovirus expression vector.Virology 1991;182: 229-238.

15. Sánchez-Martínez D, Schmid DS,W hittington W, Brown D, Reeves $W C, C$ hatterjee $S$ et al. Evaluation of a test based on baculovirusexpressed glycoprotein $\mathrm{G}$ for detection of herpes simplex virus typespecific antibodies. J Infect D is 1991;164: 1196-1199.

16. Bronfman M, Guiscafré H, Castro V, Castro R, Gutiérrez G. Medición de la desigualdad: una estrategia metodológica, análisis de las características socioeconómicas. Arch Invest Med 1988;19: 351-360.
17. Hernández-Girón C, U ribe-Salas F, C onde-G onzález C, Cruz-Valdez A, Juárez-Figueroa L, U ribe-Zúniga Patricia et al. Seroprevalencias a diversos virus y características sociodemográficas en mujeres que buscan detectarse VIH. Rev Invest Clin 1997; 49: 5-13.

18. U ribe-Salas F, Hernández-A vila $\mathrm{M}, \mathrm{C}$ onde-G lez CJ, Juárez-Figueroa L, Allen $B$, A naya- $O$ campo $R$ et al. Low HIV and STD prevalence in a random sample of female commercial sex workers in Mexico City.Am J Public Health 1997; 87: 1012-1015.

19. Lazcano-Ponce E, Smith J, Muñoz N , Conde-G lez CJ, Juárez-Figueroa $\mathrm{L}, \mathrm{Cruz} A$ et al. High prevalence of antibodies to herpes simplex virus type 2 among middle-aged women from Mexico City:A population based study. Sex Transm D is 2001; 28: 270-276.

20. van de Laar MJ,Termorshuizen F, Slomka MJ, van D oornum GJ, 0 ssewaarde JM, Brown DW et al. Prevalence and correlates of herpes simplex virus type 2 infection: Evaluation of behavioural risk factors. Int J Epidemiol 1998; 27: 127-134.

21. van Benthem BHB, Spaargaren J, van den Hoek JAR, Merks J, Coutinho RA, Prins M. Prevalence and risk factors for HSV-1 and HSV-2 antibodies in European HIV infected women. Sex Transm Infect 2001; 77: 120-124.

22. Fleming DT, McQ uillan $G M$, Johnson RE, N ahmias AJ, Aral SO, Lee FK et al. Herpes simplex virus type 2 in the United States, 1976 to 1994. N Engl J Med 1997; 337: 1105-111.

23. Safrin S, A rvin A, Mills J,A shley R. C omparison of W estern immunoblot assay and a glicoprotein $G$ enzyme immunoassay for detection of serum antibodies to herpes simplex virus type 2 in patients with AIDS.J Clin Microbiol 1992; 30: 1312-1314.

24. Ashley RL,W ald A. G enital herpes: Review of the epidemic and potential use of type-specific serology. Clin Microbiol Rev 1999; 12: 1-8. 25. U ribe-Salas F, Hernández-Girón C, C onde-G onzález C, C ruz-Valdez A, Juárez-Figueroa L, H ernández-Avila M. C aracterísticas relacionadas con ETS/VIH de hombres que trabajan en bares de la ciudad de México donde se ejerce la prostitución femenina. Salud Publica Mex 1995; 37 : 385-393.

26. U ribe-Salas F, C onde-G lez CJ, Juárez-Figueroa L, H ernández A. Sociodemographic dynamics and sexually transmitted infections in female sex workers at the Mexican-Guatemalan border. Sex Transm D is 2003; 30: 266-271

27. A braham CD, Conde-G lez CJ, Cruz-Valdez A, Zamorano-Sánchez L, Hernández-Márquez $C$, Lazcano-Ponce $E$. Sexual and demographic risk factors for herpes simplex virus type 2 according to schooling level among Mexican youths. Sex Transm D is 2003; 30: 549-555. 28. Sánchez-Alemán M, U ribe-Salas F, C onde-G lez C. High risk sexual behavior and herpes simplex type 2 infection in Mexican college students. Int J STD AID S 2002; 13 (Suppl): 45-46.

29. W orld Health 0 rganization. Estimation of the incidence and prevalence of sexually transmitted infections. Report of aW HO consultation; 2002 February 27-March 1;Treviso, Italy. D isponible en: www.who.int/hiv/pub/whohiv200214.pdf. 